\title{
Research on the Applications of Genetic Algorithm and Fuzzy Clustering Methodology for the Enhancement of Computer Network Reliability
}

\author{
Rong Wang ${ }^{1}$ \\ ${ }^{1}$ Hubei University of Science and Technology, \\ Xianning, Hubei Province,437100 China
}

\begin{abstract}
In this paper, we research on the applications of genetic algorithm and fuzzy clustering methodology for the enhancement of computer network reliability. With the coming of information age, the application of computer network and its influence scope expands unceasingly, in order to ensure the reliability of computer network operation, the negative interference from the outside world, enhance the computer network reliability become the focus of widespread attention from all walks of life. Our method performs well compared with other state-of-the-art algorithms. In the recent future, we plan to conduct more related research to polish the method.
\end{abstract}

Keywords: Genetic Algorithm; Fuzzy Clustering; Computer Network Reliability; Optimization.

\section{Introduction}

The concept of computer network is a multiple areas of different locations of the computer connected to the functional external devices effectively, thus formed a certain scale of the network system, can realize multiple computers transmit data through this system, the output of the computer information, computer data file sharing and so on. That is to say, the computer network is to use today's communication network will be set multiple machines and equipment, the formation of the unity of the computer, the equipment, in the development of today's society, computer network for all areas of development have a certain role in promoting. With the coming of information age, the application of

\author{
Bin Rao ${ }^{1, *}$ \\ ${ }^{1}$ Hubei University of Science and Technology, \\ Xianning, Hubei Province,437100 China \\ ${ }^{*}$ Corresponding Author: Bin Rao
}

computer network and its influence scope expands unceasingly, in order to ensure the reliability of computer network operation, the negative interference from the outside world, enhance the computer network reliability become the focus of widespread attention from all walks of life [1-3]. The reliability of the so-called computer network and mainly refers to the established range of conditions and the design time, the energy of the computer network system to maintain normal operation. Analysis of the computer network reliability design criteria, explore some effective measures to improve the computer network reliability has an important value. Computer network not only spread in various enterprises, after the Internet popularization, the computer users also gradually increasing every year, the computer has become an important tool in people's family, through the computer network we can deal with a lot of things for people which has the special meaning.

Once computer network appear problem, will cause great negative impact to related applications, from a certain extent, the application effect is directly affected by the reliability of the computer network, to ensure the efficient operation of computer network is an important foundation to properly solve the problem of the reliability of the computer network. The computer network reliability design criteria can be roughly summed up in the following aspects. (1) To adoption of new technology of comprehensive considerations. Improve the reliability of computer network, on the one hand, need to backbone network technology in the future for a period of 
comprehensive analysis, the developing trend of moderation has adopted some advanced technology and equipment, so that can keep up with the future computer network for a period of technology development pace, as far as possible to prolong the period of system, prevent eliminated in a short period of time situation, to better meet the real needs of business development; On the other hand, also pay attention to the inheritance system, the selection of the new technology be careful, pay attention to the effective control of risk, to ensure the compatibility of the computer network design and scalable, guarantees a smooth upgrade for the computer network. (2) Starting from the reality, adjust the quality excellent, good word-of-mouth network products, not only that, but also in accordance with the provisions of the computer network specification, according to the current domestic and foreign standards related devices and subsystems for choice, the choice of the computer network to ensure that product completely conform to the requirements of the reliability design. (3) To further improve in order to ensure the reliability of the computer network, the need for the whole life within the scope of the relevant and expenses comprehensive consideration, as far as possible to the network system of cost control, to control the operation and maintenance costs low and ensuring that the system performance to achieve the most.

To solve the mentioned issues, in this paper, we research on the applications of genetic algorithm and fuzzy clustering methodology for the enhancement of computer network reliability. In the information system of the whole unit, was the most basic is a computer network, and with the aid of computer network, can realize effective transmission of the data and voice, in addition, the computer network and the user's current and future a period of time within the unit information level and the effect of network application system has a certain relationship between, therefore, in the computer network design, as far as possible to ensure that the computer network reliability and economy. In the figure one, we illustrate the topology of the computer network structure. In the following sections, we will discuss the issues and problems in detail.
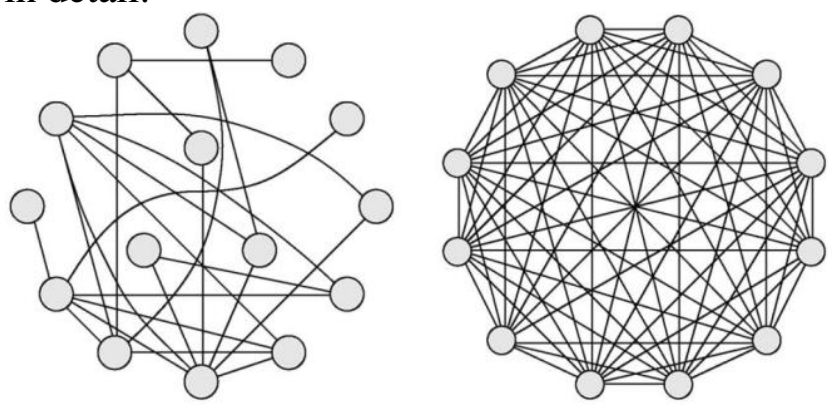

Fig. 1The Popular Topology of the Computer Network Structure

\section{The Proposed Genetic Algorithm and Fuzzy Clustering Methodology}

The Concepts and Principles of Genetic Algorithm. Genetic algorithm is a kind of based on the biological natural selection and genetic mechanism of random search algorithm, and through the group search strategy and information exchange between individuals in the search for the most advantages in solution space. Genetic algorithm has great potential in solving complex optimization problems. General algorithm possible starting from the problem of a single solution, but the genetic algorithm starts from the solution set of string search, it reflects the advanced than the traditional algorithm of genetic algorithm, the traditional optimization algorithms usually begins with a single initial value of iteration for the optimal solution, this algorithm has an obvious weakness is can't consider the global. And the genetic algorithm can well consider global, comprehensive and effective choice to choose the optimal solution.

Genetic algorithm in dealing with problems can handle more than one in the group at the same time, this also is in order to avoid the pitfalls of traditional optimization algorithm, avoid falling into local optimal solution, can realize the algorithm itself parallel at the same time. The biggest characteristic of genetic 
algorithm is it basically without the knowledge of the search space and other auxiliary information, and only use to assess individual fitness function value, on the basis of the genetic operation. The fitness function can be continuously differentiable constraints, and its domain is unfettered, can be arbitrarily set. This feature makes the genetic algorithm of genetic algorithm is widely used in the application. The proper function for the algorithm is shown in the formula one.

$$
f(x)=(x-1) /(P o P-\text { size }-1)
$$

Using wheel choice, its basic idea is each gene selection probability shown in the following.

$$
P_{k}=f_{k} / \sum_{j=1}^{p_{-} \text {size }} f_{j}+f_{k} / \sum_{i=1}^{p_{-} \text {size }} f_{i}
$$

Crossover operation above the connectedness of network as much as possible, but is likely to produce do not represent the correct network connecting structure. So it is necessary to use adjustment algorithm to solve this kind of contradiction. In this kind of computer network optimization, we are doing this is not very appropriate, because the choice of route and link capacity is to determine the choice of related, so the best solution is to consider these two problems at the same time. We can see clearly the constraint condition is a complex nonlinear programming problem, for this kind of problem, we could be based on the genetic algorithm, design a genetic optimization algorithm. Genetic by computer numerical examples show that this algorithm can calculate the approximate optimal solution, and the precision is higher. Genetic algorithm is a reference to the evolution of the biological laws to simulate the operation, so the algorithm is generally don't have to determine a specific calculation rule, but according to the change rules of probability to guide the search direction of the algorithm. A series of characteristics that genetic algorithm is used in computer network.
The Core Techniques of Fuzzy Clustering. Clustering is an unsupervised learning method, which aims to a target data set are divided into several classes. Division is based on the similarity between the data centralized data or the opposite sex. Fuzzy clustering and prototype index according to the relationship between represented with fuzzy membership degree. Fuzzy clustering to avoid the rigid clustering algorithm in data can only be the only belongs to one kind of faults. Outliers mining are one of the basic tasks of data mining, the current received widespread attention. In many cases, the rare event is more interesting than a normal event, such as the abnormal fluctuations of parameters in complex industrial process, etc. Outliers in data mining research and outliers itself become the focus. FCPM algorithm stresses from crumb structure to the feedback information from the data set. In this algorithm, assuming that the data points is produced according to the crumb structure and the membership degree not only represent data points belonging to the proportion of each cluster center, at the same time, data points membership degree can also be interpreted as belonging to that part of each cluster center. Early research focused on misuse detection of intrusion detection technology, through monitoring the target system of the specific behavior and the definition of the known beforehand invasive pattern matching to determine whether the behavior, is of high accuracy, stability, detection effect and fast response speed, low rate of false positives, but can't find unknown attack, attack feature library need to constantly update, this is still a difficult problem in cloud computing era. Anomaly detection is based on the normal work mode of protected target, will deviate from the normal behavior of the network data connection as aggression, can find unknown attack, but had a higher rate of false positives, the formula three shows this feature. 


\section{(3)}

$$
S p t=\min _{i=k}\left\|v_{i}-v_{k}\right\|^{2}
$$

Collaborative coefficient should be determined according to experience and need a lot of experiment suitable value. When the data feature selection, the number of feature subset and the determination of each group of features in the subset, affect the test results shown as the follows.

$$
C m p=\sum_{j=1}^{n} \sum_{i=1}^{c} u_{i j}^{m}\left(x_{j}-v_{i}\right)^{2}
$$

\section{The Enhancement of Computer Network} Reliability. The development of computer network is extremely large, and also on a more complex, made up of multiple vendors, and the development of the network structure is developed by different manufacturers, with a strong comprehensive. Under this development situation, however, the computer network operation parameters and lack of standardization and management, and also used in computer network software update faster, in this kind of complex network structure, software update and management is bound to cause a certain complexity. Dual network structure design of computer network, from the factors of computer network technology get promoted, in a sense, dual network structure design is to add a layer of backup network computer network operation, and then combine today's computer redundancy technology to improve the fault tolerance of the computer network, the operation of the computer network reliability has a major role. Traditional computer network in data transmission, backup, because of low reliability, will cause some difficulties, and through the double forms of network structure, by brand-new network internal structure to realize synchronous transmission and backup of computer network information data. Computer network fault tolerance is a security of computer network operation in traditional computer network operation, once the network problems will continue to delay the user operation, the effects of the great. Computer network is a country to carry out the modernization important basic facilities, is an effective way of enterprise information construction. For modern computer networks, the scale is more and more big, the heterogeneous degree also increase, more should pay attention to the reliability of the computer network, pay attention to in practice to improve the computer network reliability design principles to sum up, the construction of high reliability of the computer network play a role of guidance, to ensure that the network operation during the negative influence from the outside world, effectively avoid the lines or partial failure caused by the comprehensive computer network paralysis condition. To safeguard the computer network security effectively, is going to build a good network environment, this will ensure the security of network computer room environment which will be meaningful.

\section{Experiment and Simulation}

In addition to the reality of the actual system has the typical structure such as series, parallel, beside, there are many typical structures cannot be said of large and complex system. Contact adjacency matrix and matrix method, is essentially on a matrix multiplication and multiplication for many times, when the network node, then contact matrix method is suitable for hand calculation. Computer network system reliability in terms of solution actual problem the necessity of the research, from the point of view of theory there are also many problems are discussed. In the figure two, we demonstrate experimental simulation of proposed methodology. 

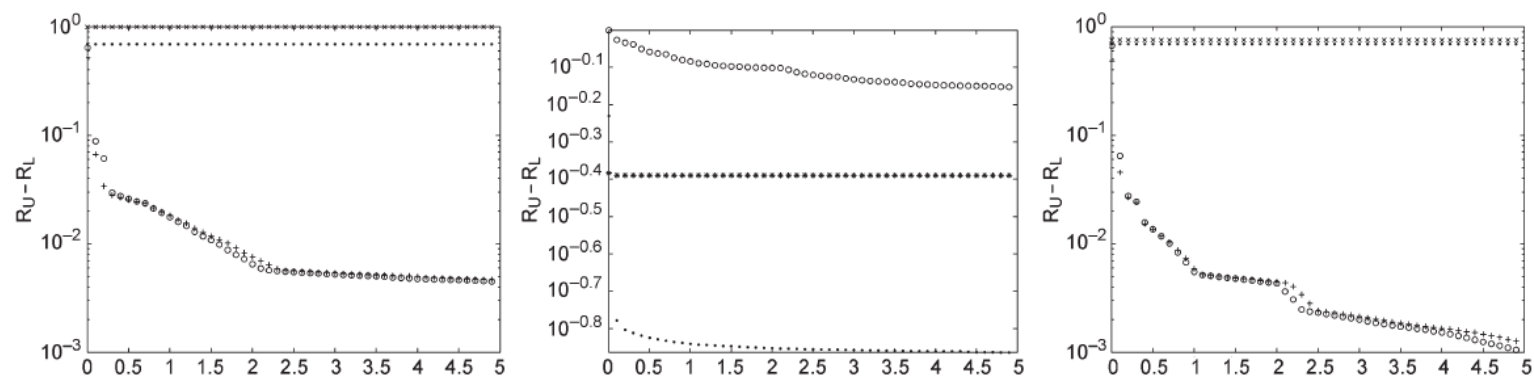

Fig. 2The Experimental Analysis of the Proposed Methodology

\section{Conclusion}

In this paper, we research on the applications of genetic algorithm and fuzzy clustering methodology for the enhancement of computer network reliability. Computer network is the geographical position is different, has an independent function of the computer with the help of a data communication system and network software to connect, make its can be smoothly between the data communication and resource sharing of computer system, computer network is the product of the combination of computer technology and communication technology. In the operation process of computer network, the host subsystem will have a series of data in and out in succession. The experimental simulation proves the effectiveness and feasibility of the proposed methodology.

\section{Acknowledgement}

The scientific research project of Hubei science and Technology Institute,Number: ky14055

\section{References}

[1] Huang, Y., \& Shen, W. Q. (2014). Management and optimization of reliability of network technology. Computer Development \& Applications.

[2] Chen H Y. The Research of Computer Complex Network Reliability Evaluation Method Based on GABP Algorithm[J]. Applied Mechanics \& Materials, 2014, 556-562.

[3] Shao H, Dong L, Wu J. Research on multi-criterion optimization of computer-communication network reliability-using genetic algorithms[J]. Journal of Chemical \& Pharmaceutical Research. 